EPJ Web of Conferences 13, 03002 (2011)

DOI: $10.1051 /$ epjconf/20111303002

(C) Owned by the authors, published by EDP Sciences, 2011

\title{
Measurements of hadron production at CMS
}

Ferenc Siklér,a on behalf of the CMS Collaboration

KFKI Research Institute for Particle and Nuclear Physics, Budapest, Hungary

\begin{abstract}
First results on hadron production using the $0.9,2.36$ and $7 \mathrm{TeV}$ data are presented. The topics covered include spectra and multiplicity distributions of charged hadrons, spectra of strange hadrons, angular and Bose-Einstein correlations of charged particles. This is just the beginning of a successful physics program at the LHC, with possible future discoveries.
\end{abstract}

\section{Introduction}

The Compact Muon Solenoid (CMS) experiment detected the first proton-proton collisions at the end of November in 2009. During the 2009 run more than 300000 minimum bias events were collected at $\sqrt{s}=0.9 \mathrm{TeV}$ and about 20000 at $2.36 \mathrm{TeV}$. In the early spring of 2010 the experiment was collecting minimum bias triggers, with typical rates of 50-100 Hz. Having observed the first $7 \mathrm{TeV}$ collisions on March 30th (Fig. 1), there have been several million collisions recorded so far, which resulted in many interesting physics results.

This report shows the detector and reconstruction performance, as well as the first physics results from inclusive hadron production.

\section{Detector}

A detailed description of the CMS experiment can be found elsewhere [1]. The central feature of the CMS apparatus is a superconducting solenoid, of $6 \mathrm{~m}$ internal diameter. Within the $B=3.8 \mathrm{~T}$ field volume are the silicon pixel

a e-mail: sikler@rmki.kfki.hu

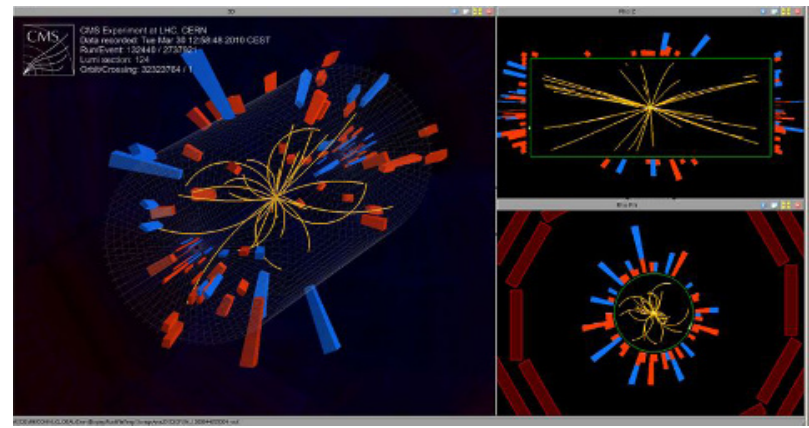

Fig. 1. A reconstructed $7 \mathrm{TeV}$ proton-proton event with tracks and calorimeter towers. and strip trackers, the crystal electromagnetic calorimeter (ECAL) and the brass-scintillator hadronic calorimeter (HCAL). Muons are measured in gaseous detectors embedded in the iron return yoke. Besides the barrel and endcap detectors, CMS has extensive forward calorimetry.

Mid-rapidity charged particles are tracked by three layers of silicon pixel detectors, made of 66 million $100 \times$ $150 \mu \mathrm{m}^{2}$ pixels, followed by ten microstrip layers, with strips of pitch between 80 and $180 \mu \mathrm{m}$. The silicon tracker provides the vertex position with $\sim 15 \mu \mathrm{m}$ accuracy, and an excellent momentum resolution.

The ECAL has an energy resolution of better than $0.5 \%$ above $100 \mathrm{GeV}$. The HCAL, when combined with ECAL, measures jets with a resolution $\Delta E / E \approx 100 \% / \sqrt{E} \oplus 5 \%$. The calorimeter cells are grouped in projective towers, of granularity $\Delta \eta \times \Delta \phi=0.087 \times 0.087$ at central rapidities and $0.175 \times 0.175$ at forward rapidities. The lead-tungstate crystals are $25.8 X_{0}$ thick in the barrel and $24.7 X_{0}$ thick in the end-caps.

The CMS apparatus has an overall length of $22 \mathrm{~m}$, a diameter of $15 \mathrm{~m}$, and weighs 12500 tonnes.

\section{Detector performance}

The invariant mass distributions of photon pairs detected in ECAL barrel show nice peaks of $\pi^{0}$ and $\eta$. Their positions and the ratio of signal to background prove a good agreement between data and simulation. The energy scale is also seen to agree within $2 \%$. Electrons are reconstructed and identified with the combination of tracker and ECAL information. The observed $p_{T}$ spectrum shows a good agreement with simulation [2]. Photon conversions are also reconstructed, and $\pi^{0}$ decays where one photon was converted in the tracker have been observed [2].

The performance of the tracker is demonstrated with the help of long lived resonances, decaying to charged hadrons off the primary interaction vertex [3]. In Fig. 2] invariant mass spectra of $\pi^{+} \pi^{-}$pairs, as well as and $\mathrm{p} \pi^{-}\left(\overline{\mathrm{p}} \pi^{+}\right)$ pairs are shown with fits to signal and background. The obtained fit parameters match very well the mass of $\mathrm{K}_{\mathrm{S}}^{0}$ and

This is an Open Access article distributed under the terms of the Creative Commons Attribution-Noncommercial License 3.0, which permits unrestricted use, distribution, and reproduction in any noncommercial medium, provided the original work is properly cited. 

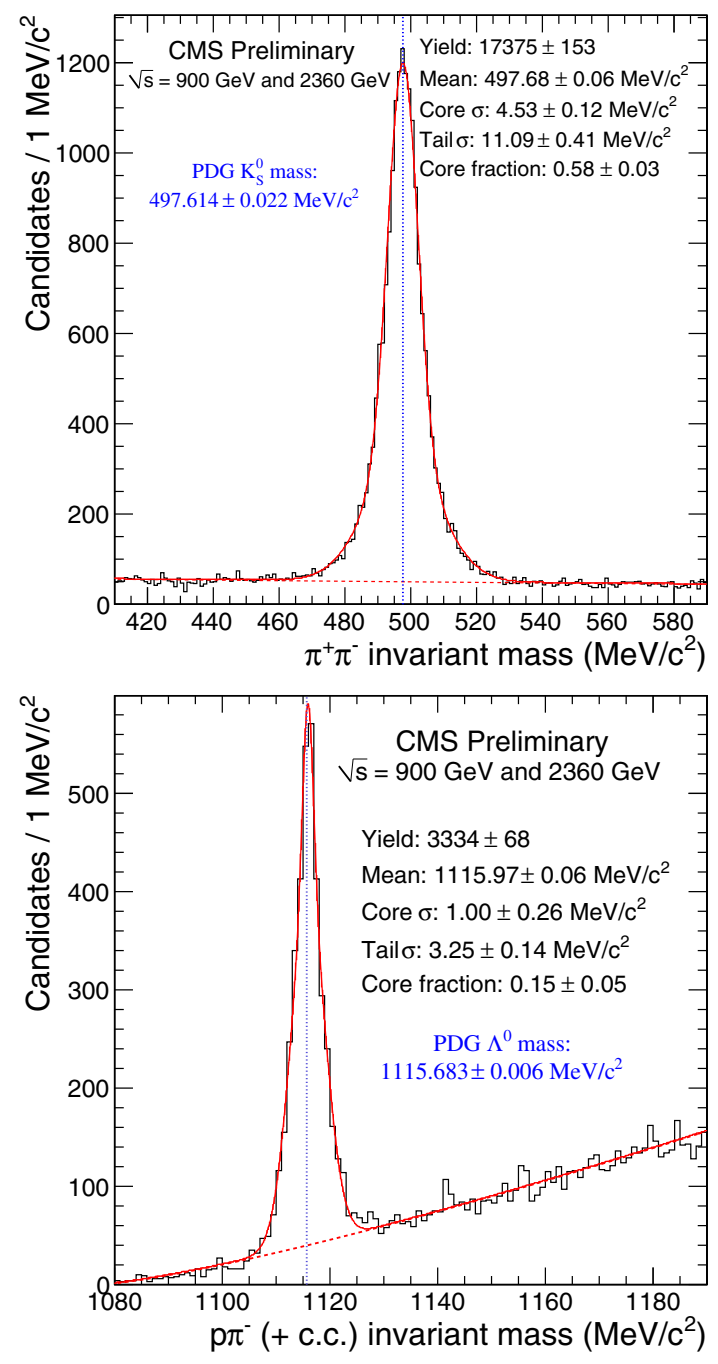

Fig. 2. Top: Fitted $\pi^{+} \pi^{-}$mass spectrum for data. Bottom: Fitted $\mathrm{p} \pi^{-}(+$charge conjugate) mass spectrum for data. Uncertainties shown are statistical only.

$\Lambda$, while the signal width and the shape of the background agree with simulation results. The identified resonances can be further combined with other charged hadrons to reach higher mass states, such as the on-vertex $\mathrm{K}^{*}(892)$ and the $\Xi^{ \pm}$. The mass peaks of both particles show an excellent agreement with the PDG values.

The tracker can be used for particle identification at low momentum, since the energy deposited in the silicon layers allows to measure the energy loss rate of charged particles (Fig. 3-top), in this case with a power mean estimator [3]. That capability is readily used to enhance kaons in order to facilitate the yield extraction of the $\phi(1020)$ meson (Fig. 3-bottom).

In CMS jets can be reconstructed in three ways [4]: using calorimeters only; using energy depositions in the calorimeters with track corrections; using full reconstructed particles (particle flow [5]). The measured and the corresponding simulated kinematic distributions for the three methods show a nice agreement [4]. The measurement of
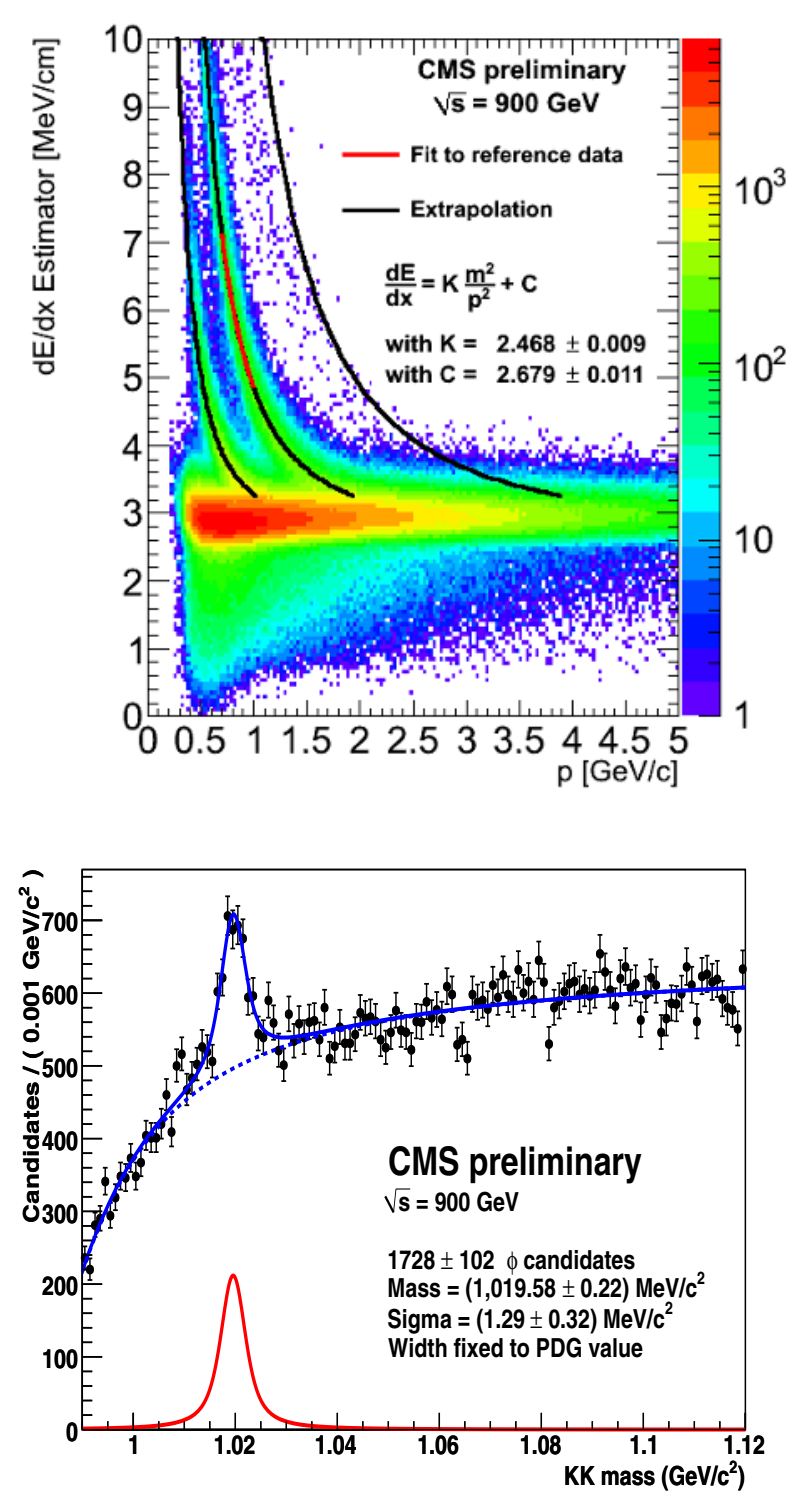

Fig. 3. Top: $d E / d x$ versus $p$ in data collected at $0.9 \mathrm{TeV}$, during the December 2009 run. The red line gives a fit with proton mass assumption, in a restricted $p$ range, while black lines show extrapolations. Bottom: Fit to the mass distribution of kaon candidate pairs in data. The natural width is fixed to the value of $\Gamma_{\phi(1020)}=$ 4.26 MeV/c $c^{2}$.

the missing transverse energy proved to be robust over the full data-taking period [6]. Nevertheless very high missing $E_{T}$ values were also detected. They were carefully studied and traced back to anomalous noise in HCAL (functioning of hybrid photo diodes, photo-multiplier window hits), in ECAL (single hit crystals, "spikes") and also due to beamhalo muons. A very efficient cleaning procedure was introduced that reduced the missing $E_{T}$ tail [6]. 

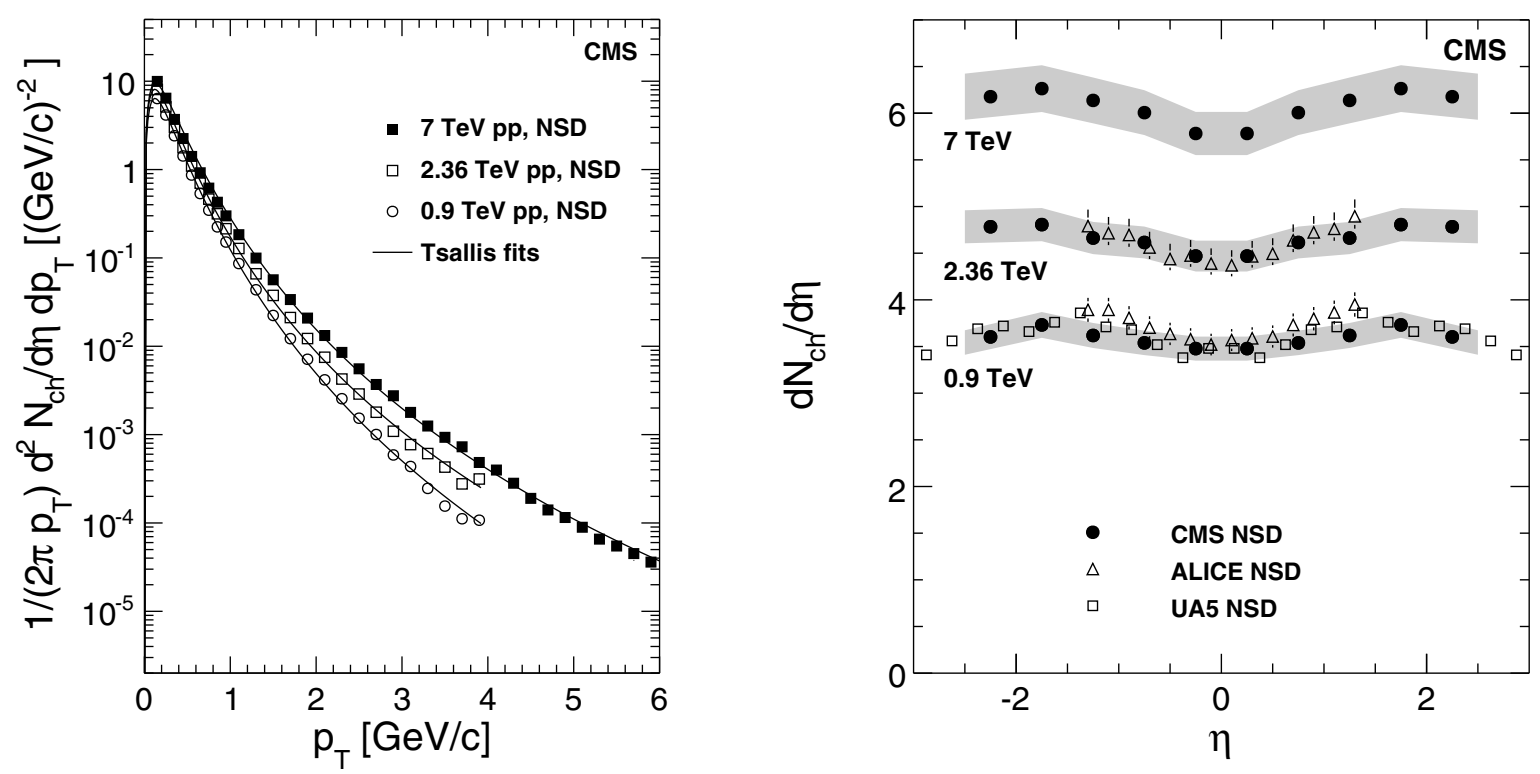

Fig. 4. Left: Measured yield of charged hadrons for $|\eta|<2.4$ with systematic uncertainties (symbols), fit with a Tsallis-type function. Right: Reconstructed $d N_{c h} / d \eta$ distributions averaged over the cluster counting, tracklet and tracking methods from CMS (filled circles), compared to data from UA5 and ALICE experiments where available. The shaded band represents systematic uncertainties of the CMS measurement.

\section{Physics results}

In the following results on soft QCD observables are described.

\subsection{Spectra of charged hadrons}

The measurement of charged hadron spectra is important since it provides basic quantities for tuning soft particle production models. It also constitutes to the background of more exclusive processes. In CMS the two-sided scintillator based minimum bias trigger is most sensitive to nonsingle-diffractive (NSD) collisions, hence all results below were corrected to NSD events. The spectra of hadrons is determined with the pixel and silicon tracker, using three different reconstruction methods: pixel hit counting, tracklets, and full tracks [7,8].

In case of hit counting the number of track hits, clusters, are counted in the barrel region of the pixel detector. With the previously determined collision vertex the pseudorapidity $\eta$ is calculated for each hit and histogrammed. This very simple method is immune to detector mis-alignment but it needs several corrections due to looping low- $p_{T}$ particles, resonance decays, $\delta$-electrons, nuclear interactions. It is sensitive even to very low $p_{T}$ particles, of about $30 \mathrm{MeV} / c$. Tracklets are formed out of hit pairs in the pixel detector which are compatible with each other in $\eta$ and azimuth directions. This method has lower background than the pixel hit counting, supplemented by a data-driven background subtraction (side-band method). The full tracking method uses all pixel and strip hits associated with the charged particle. It provides $\eta$ and $p_{T}$ at the same time. This approach is the most complex but also the most sensitive one.

The measured $p_{T}$ spectra of charged hadrons at $\sqrt{s}=$ 0.9, 2.36 and $7 \mathrm{TeV}$ in NSD events are shown in Fig. 4.left. A Tsallis-type fit is also displayed: this function successfully combines and describes the low $p_{T}$ exponential and the high $p_{T}$ power law nature of the distribution, with few parameters. The pseudorapidity-density $d N_{c h} / d \eta$ as function of $\eta$ is shown in Fig. 4 right again for all energies, with comparisons to results of other experiments.

The center-of-mass energy dependence of $\left\langle p_{T}\right\rangle$ and the $d N_{c h} / d \eta$ at $\eta=0$ is shown in Fig. 5. While the average $p_{T}$ shows the expected rise, the pseudorapidity density gives a higher than expected accelerated increase, when going from 0.9 to $7 \mathrm{TeV}$, if compared to simulation expectations.

\subsection{Spectra of strange hadrons}

Spectra of strange hadrons have been measured at 0.9 and $7 \mathrm{TeV}$ center-of-mass energies in NSD events [9]. The $\mathrm{K}_{\mathrm{S}}^{0}$, $\Lambda, \bar{\Lambda}$ and $\Xi^{ \pm}$particles were reconstructed from their decay topologies in the tracker, for details see [10].

The corrected $\mathrm{K}_{\mathrm{S}}^{0}$ and $\Lambda$ yields are reported, normalized to the number of NSD events, in Figs. 6 and 7 , respectively. The $y$ distributions are relatively flat in the central rapidity range, while slowly decreasing at higher values, especially at $\sqrt{s}=7 \mathrm{TeV}$. They show a steeply falling distribution in $p_{T}$. The solid lines in the figures are fit results 

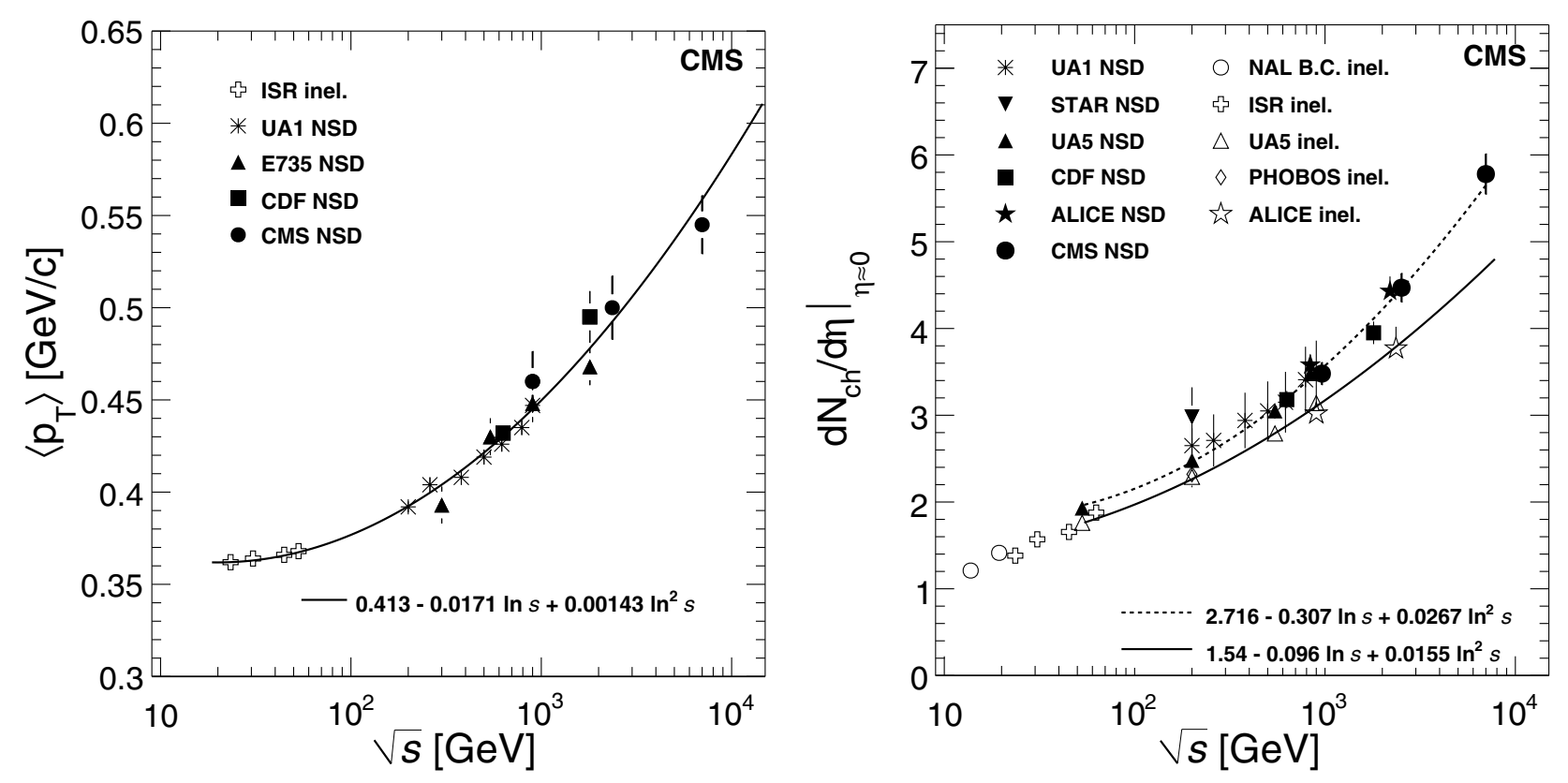

Fig. 5. Left: Energy dependence of the average transverse momentum of charged hadrons. The CMS data points are evaluated for the range $|\eta|<2.4$. The curve shows the fit to the data points. The error bars on the CMS data points include systematic uncertainties. Right: Charged-hadron pseudorapidity density in the central region as a function of center-of-mass energy in pp and $\mathrm{p} \overline{\mathrm{p}}$ collisions including lower energy data, together with various empirical parameterizations fit to the data corresponding to the inelastic (solid and dotted curves with open symbols) and to the NSD (dashed curve with solid symbols) event selection.
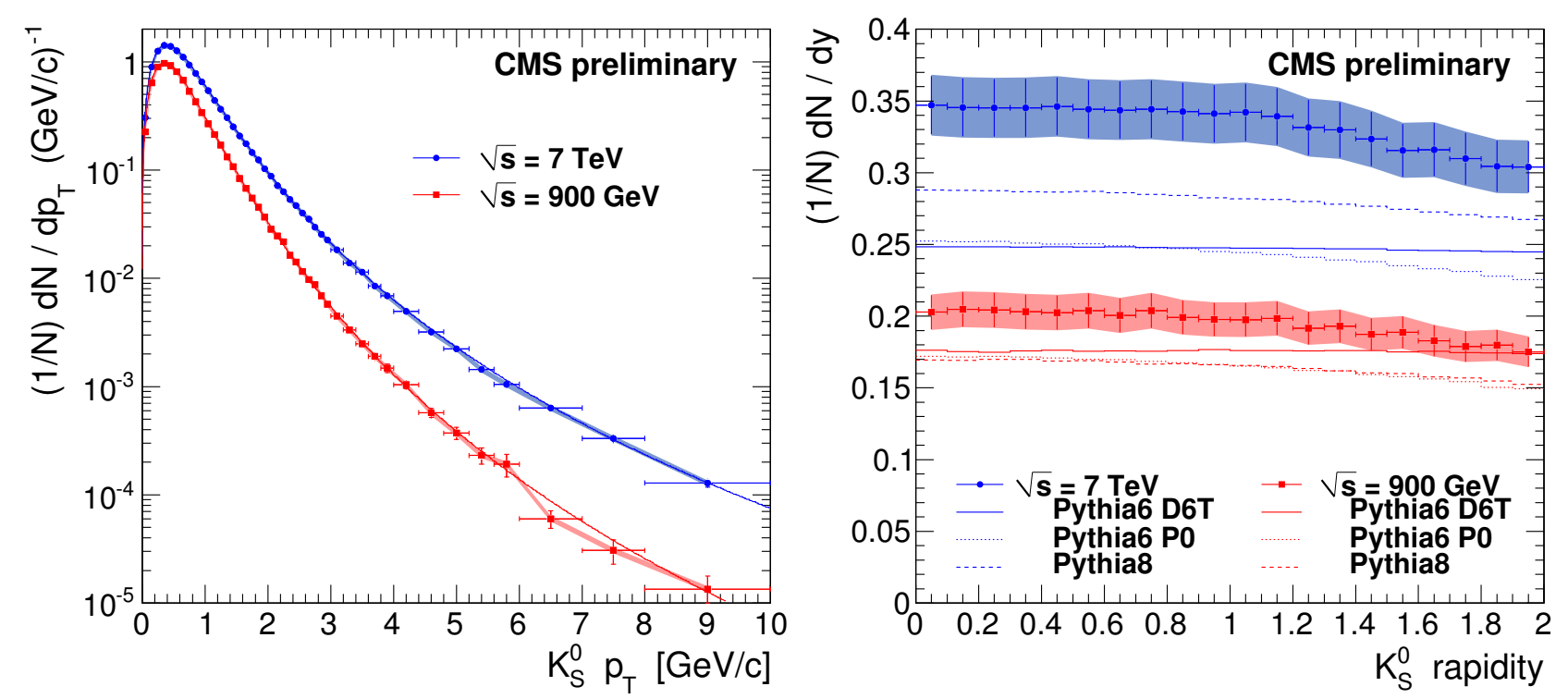

Fig. 6. $\mathrm{K}_{\mathrm{S}}^{0}$ production per NSD event versus $p_{T}$ (left) and $|y|$ (right), compared to three Pythia Monte Carlo samples. The inner vertical error bars (when visible) show the statistical errors, the outer the statistical and uncorrelated systematic uncertainties summed in quadrature. The overall normalization uncertainty is shown as a band. The solid line is a fit with the Tsallis function as described in the text. 

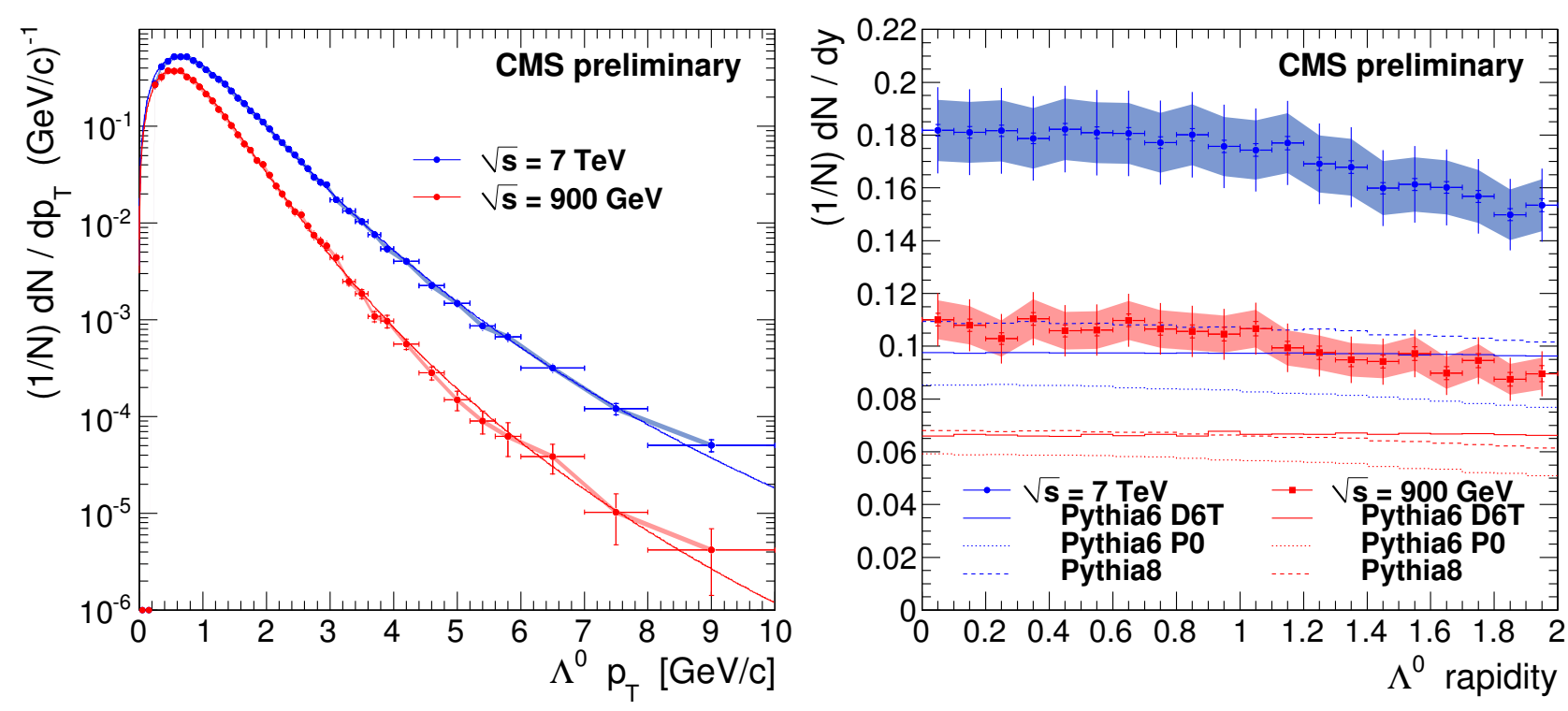

Fig. 7. $\Lambda$ production per NSD event versus $p_{T}$ (left) and $|y|$ (right), compared to three PyTHIA Monte Carlo samples. The inner vertical error bars (when visible) show the statistical errors, the outer the statistical and uncorrelated systematic uncertainties summed in quadrature. The overall normalization uncertainty is shown as a band. The solid line is a fit with the Tsallis function as described in the text.

with the Tsallis function of the form

$$
\frac{1}{N} \frac{d N}{d p_{T}}=C p_{T}\left[1+\frac{\sqrt{p_{T}^{2}+m^{2}}-m}{n T}\right]^{-n}
$$

The average $p_{T}$ was extracted using this function, yielding for $\sqrt{s}=0.9 \mathrm{TeV}(7 \mathrm{TeV}) 0.657 \pm 0.002 \pm 0.038(0.789 \pm$ $0.001 \pm 0.046 \mathrm{GeV} / c)$ for $\mathrm{K}_{\mathrm{S}}^{0}$, and $0.849 \pm 0.004 \pm 0.076$ $(1.054 \pm 0.003 \pm 0.094 \mathrm{GeV} / c)$ for $\Lambda$. As expected, the average $p_{T}$ increases with increasing particle mass and with increasing center-of-mass energy. The ratio for $\Lambda / \mathrm{K}_{\mathrm{S}}^{0}$ production is found to be $0.54 \pm 0.02$ (stat.) \pm 0.05 (syst.) at $\sqrt{s}=0.9 \mathrm{TeV}$, and $0.52 \pm 0.01$ (stat.) \pm 0.05 (syst.) at $\sqrt{s}=$ $7 \mathrm{TeV}$.

The large increase in the measured production cross section of strange particles as the center-of-mass energy increases from 0.9 to $7 \mathrm{TeV}$ is not modelled by the PyTHIA event generator [11]. The other noticeable feature is that the deficit in strange particle production as modelled in the simulation gets worse as the particle mass and strangeness content increase.

\subsection{Event-by-event multiplicity}

The charged particle multiplicity distributions of the NSDevents were measured at all center-of-mass energies [12]. A full correction of detector resolution and acceptance effects, with an extrapolation to zero transverse momentum, is used to measure the charged particle multiplicity distribution in increasing pseudorapidity domains from $|\eta|<0.5$ to $|\eta|<2$.4. They can be compared to Monte Carlo models for soft particle production (Fig. 8) and with experimental data at lower energies.

Although some models provide an adequate description of Tevatron and LEP data, none is able to describe simultaneously the multiplicity distributions and the $p_{T}$ spectrum at the highest LHC energy of $\sqrt{s}=7 \mathrm{TeV}$. In general all models produce too few low momentum particles.

The remarkable large multiplicity tail observed at $\sqrt{s}=$ $7 \mathrm{TeV}$ indicates a clear violation of KNO scaling with respect to lower energies and merits further studies (Fig. 9).

The observed rise of the mean charged particle multiplicity with increasing center-of-mass energy (not shown) is also steeper than extrapolations of fits at lower energies imply.

\subsection{Angular correlations}

CMS has measured two-particle angular correlations over a wide range in $\Delta \eta$ and $\Delta \phi$ in pp collisions at $\sqrt{s}=0.9$, 2.36 and $7 \mathrm{TeV}$ [14]. Short-range correlations are observed over the full range in $\Delta \phi$, with a maximum at $\Delta \eta=0$ which becomes wider at larger $\Delta \phi$ (Fig. 10). In the context of the cluster model the correlation function $R$ is parametrized as

$$
R(\Delta \eta)=\left(K_{e f f}-1\right)\left[\frac{\Gamma(\Delta \eta)}{B(\Delta \eta)}-1\right], \quad \Gamma(\Delta \eta) \propto \exp \left[-\frac{(\Delta \eta)^{2}}{4 \delta^{2}}\right]
$$

where the background distribution $B$ is obtained by eventmixing. The effective cluster size $K_{\text {eff }}$ and decay width $\delta$ are extracted from the two-particle pseudorapidity correlation function, and compared with previous experiments, as well as Pyтнia event generator (Fig. 11). Dependence 

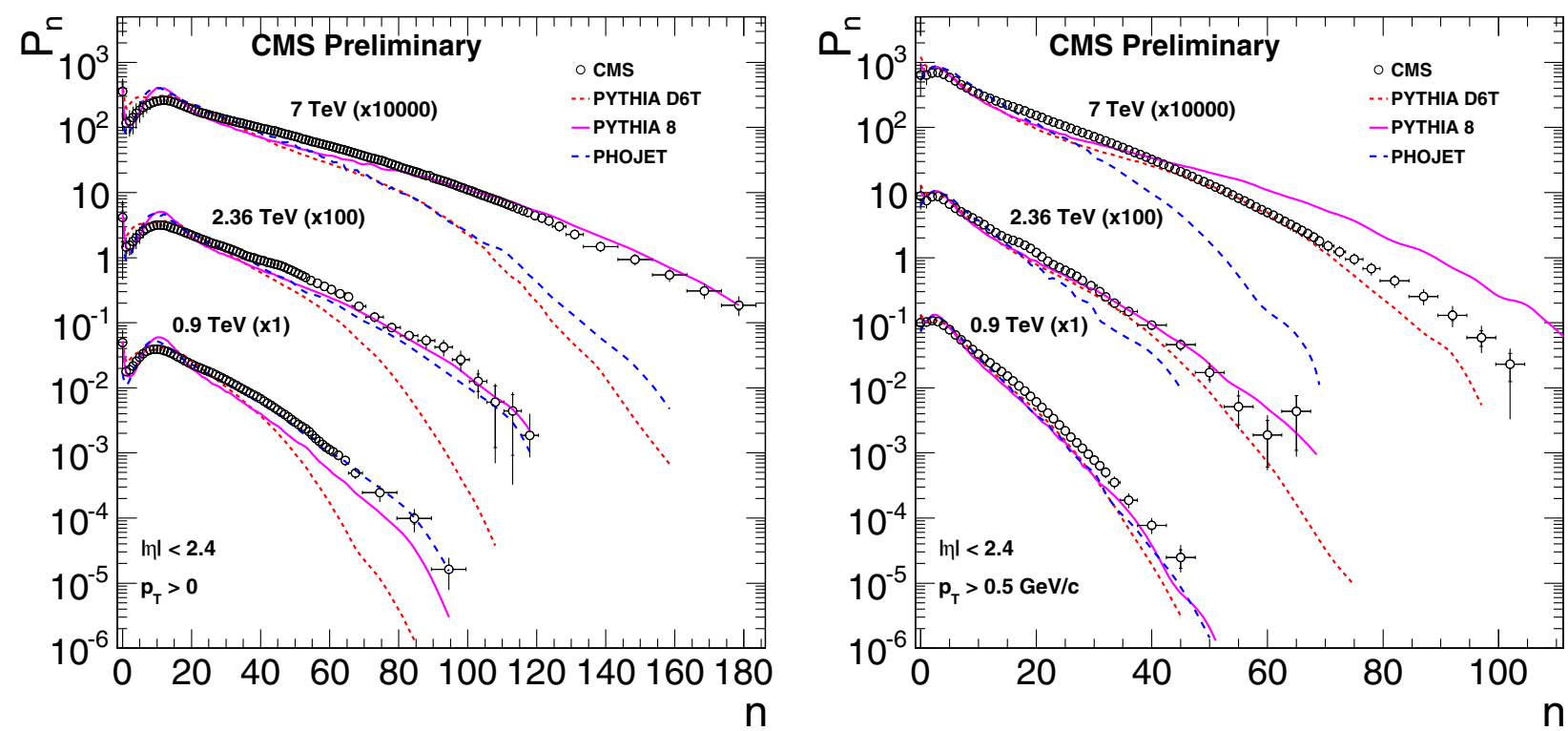

Fig. 8. The charged particle multiplicity distributions in $|\eta|<2.4$ for $p_{T}>0$ (left) and $p_{T}>500 \mathrm{MeV} / c$ (right), compared to two different Рүтніа models and the Рнолет model [13] at $\sqrt{s}=0.9,2.36$, and $7 \mathrm{TeV}$. Results for different center-of-mass energies are scaled with a multiplicative factor for clarity.
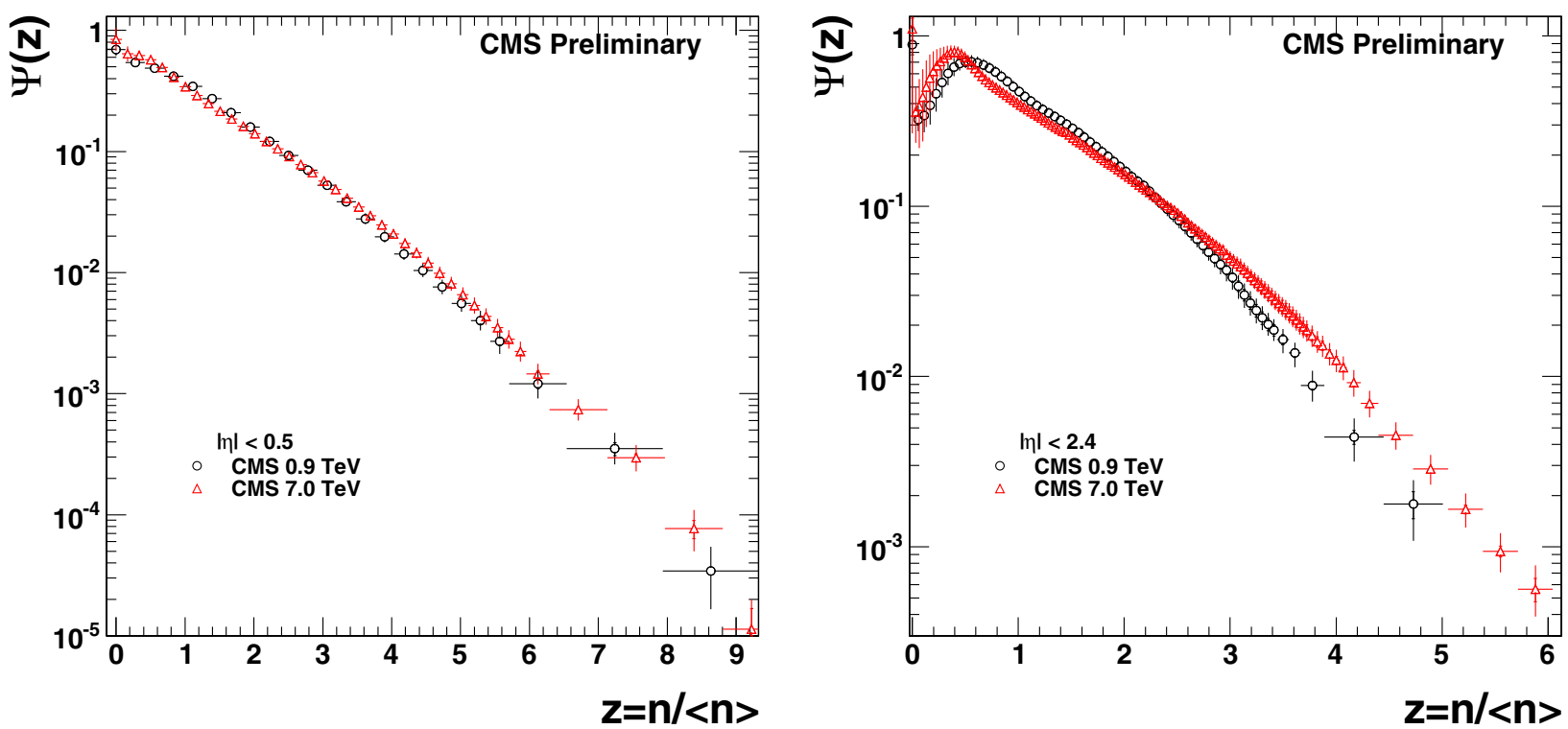

Fig. 9. The charged particle multiplicity distributions in KNO form at in two pseudorapidity intervals $|\eta|<0.5$ (left) and $|\eta|<2.4$ (right).

of the cluster size on beam energy is observed, while the cluster width is essentially constant. Cluster properties are also studied in the near side $(0<\Delta \phi<\pi / 2)$ and away side $(\pi / 2<\Delta \phi<\pi)$ regions separately, corresponding to QCD physics at different $p_{T}$ regime. The short-range correlation strength (or equivalently the effective cluster size) exceeds the expectation from the decays of resonance particles, suggesting the need for other sources of short-range correlations.
Cluster properties are strongly modified in heavy ion collisions at RHIC relative to pp collisions by the presence of a nuclear medium. Future studies in $\mathrm{pp}$ and heavy ion collisions at much higher LHC energies will further clarify the evolution of cluster properties. 

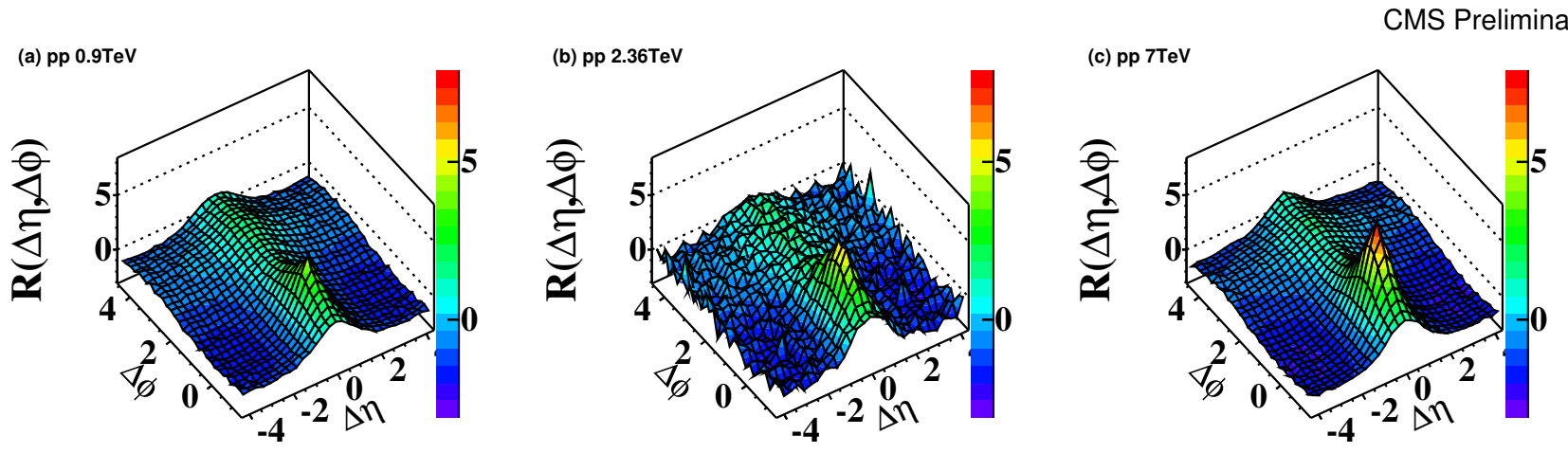

Fig. 10. Two-particle angular correlation function in $\Delta \eta$ and $\Delta \phi$ in pp collisions at 0.9 , (b) 2.36 and (c) $7 \mathrm{TeV}$.

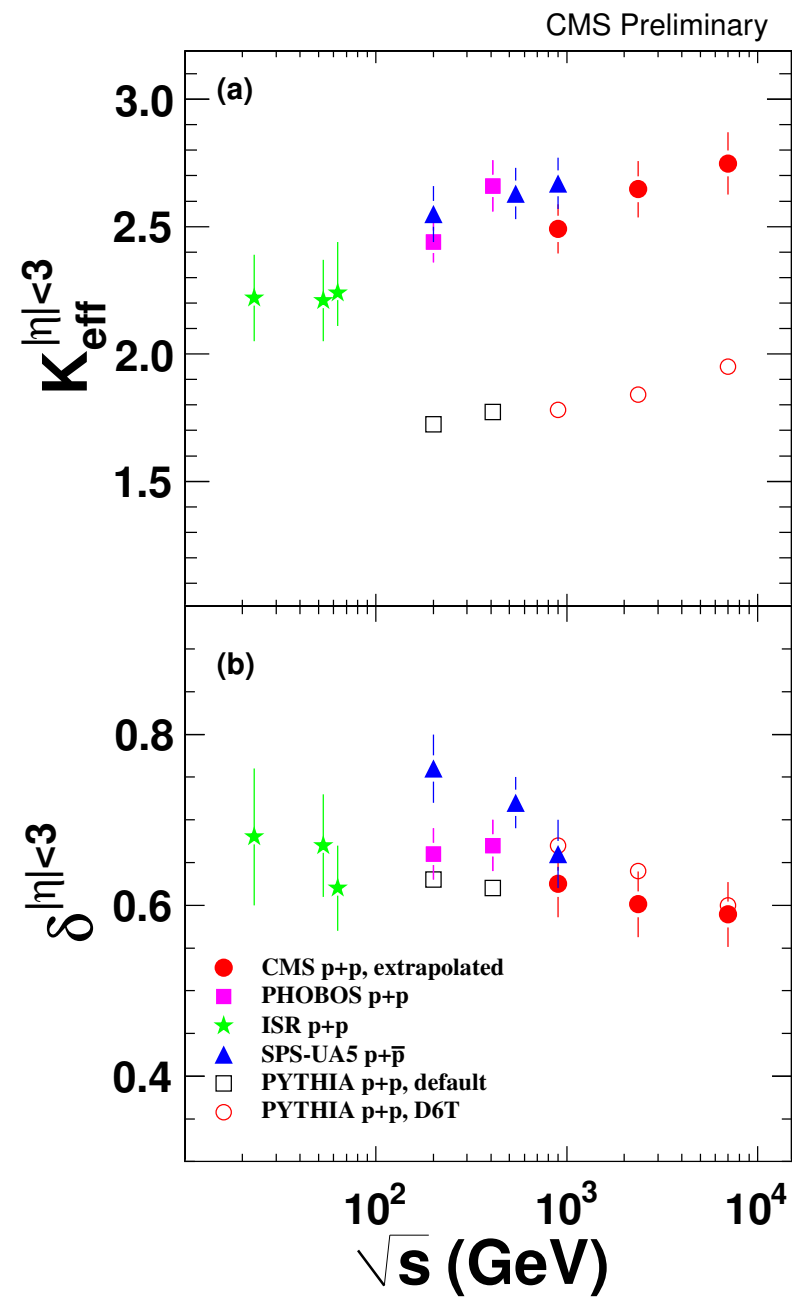

Fig. 11. (a) $K_{\text {eff }}$ and (b) $\delta$ as a function of s using a model dependent extrapolation of CMS data to $p_{T}=0$ and $|\eta|<3$ (solid circles), as well as data from other experiments. Open circles and squares show the Pyтнia results respectively.

\subsection{Bose-Einstein correlations}

The Bose-Einstein correlation is the manifestation of a quantum effect. It enhances the number of identical boson pairs with low relative momentum. By measuring the ratio of signal and uncorrelated background pairs, as a function of $Q=\sqrt{m_{i n v}^{2}-4 m_{\pi}^{2}}$, the size of the correlated emission region can be inferred. Here $m_{i n v}$ denotes the invariant mass of the boson pair assuming pion mass $m_{\pi}$. The uncorrelated reference sample is difficult to construct, here a combination of several prescriptions was used. In order to reduce the bias, a double ratio was used: it is the ratio obtained from data as described above, divided by the ratio deduced from simulation without the Bose-Einstein effect included. The ratio distribution is approximately the Fourier transform of the original spatial distribution. The distributions are shown in Fig. 12 for $\sqrt{s}=0.9 \mathrm{TeV}$ and $2.36 \mathrm{TeV}$, respectively [15].

Phenomenological parameterizations with an exponential shape fit the data significantly better than with a Gaussian shape. The parameters, radius and correlation strength, extracted from fits using an exponential form are $r=1.59 \pm$ 0.05 (stat.) \pm 0.19 (syst.) fm and $\lambda=0.625 \pm 0.021$ (stat.) \pm 0.046 (syst.) at $0.9 \mathrm{TeV}$, and $r=1.99 \pm 0.18$ (stat.) \pm 0.24 (syst.) fm and $\lambda=0.663 \pm 0.073$ (stat.) \pm 0.048 (syst.) at $2.36 \mathrm{TeV}$. An increase of the parameter $r$ with charged particle multiplicity in the event is observed (Fig. 13).

\section{Summary}

The CMS experiment and its detector systems show an excellent performance. The first publications using the 0.9 , 2.36 and $7 \mathrm{TeV}$ data are already available. This is just the beginning of a successful physics program at the LHC, with possible future discoveries.

\section{Acknowledgments}

The author wishes to thank to the Hungarian Scientific Research Fund (K 81614, NK 81447), the National Office for 


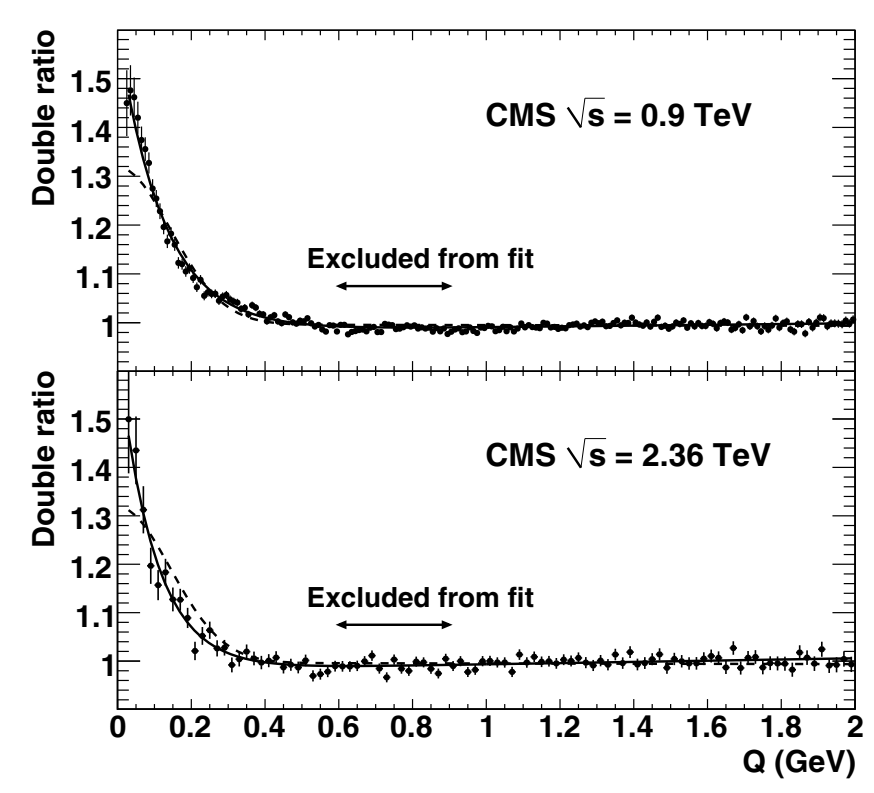

Fig. 12. Distributions of the two-particle correlation function for charged hadrons at $\sqrt{s}=0.9 \mathrm{TeV}$ (top) and $2.36 \mathrm{TeV}$ (bottom) center-of-mass energies. Measured points (symbols) and the corresponding exponential fits are shown (solid curves).

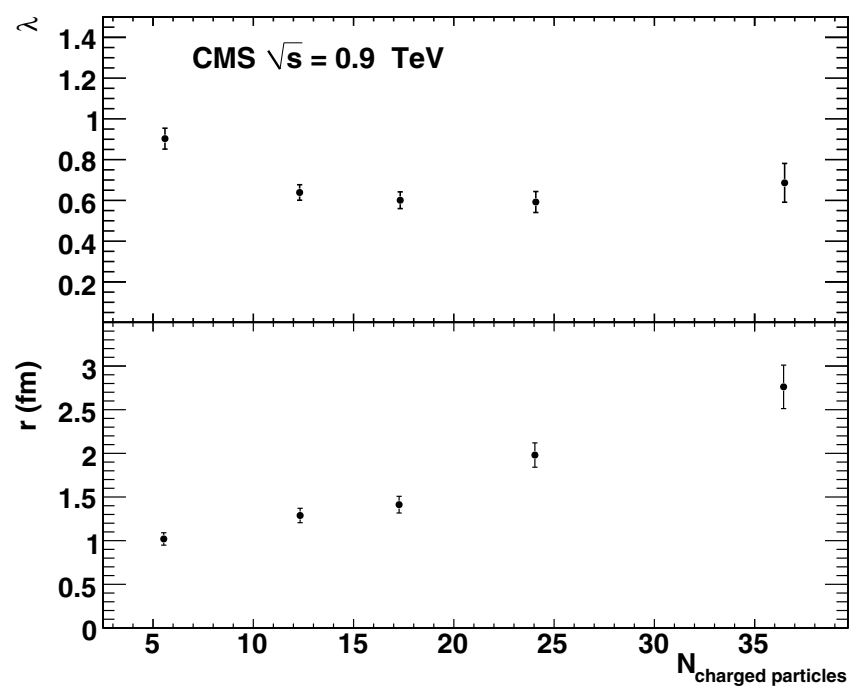

Fig. 13. Values of the $\lambda$ and $r$ parameters as a function of the charged particle multiplicity in the event. The errors are statistical only.

Research and Technology (H07-B 74296), and the Swiss National Science Foundation (128079) for their support.

\section{References}

1. R. Adolphi et al. [CMS Collaboration], JINST 3 (2008) S08004.
2. CMS Collaboration, CMS PAS EGM-10-001.

3. CMS Collaboration, CMS PAS TRK-10-001.

4. CMS Collaboration, CMS PAS JME-10-001.

5. CMS Collaboration, CMS PAS PFT-10-001.

6. CMS Collaboration, CMS PAS JME-10-002.

7. V. Khachatryan et al. [CMS Collaboration], JHEP 02 (2010) 041 [doi:10.1007/JHEP02(2010)041].

8. V. Khachatryan et al. [CMS Collaboration], Phys. Rev. Lett. 105 (2010) 022002 [doi:10.1103/PhysRevLett.105.022002].

9. CMS Collaboration, CMS PAS QCD-10-007.

10. K. Krajczár et al. [CMS Collaboration], in these proceedings.

11. T. Sjostrand, S. Mrenna and P. Z. Skands, JHEP 0605 (2006) 026 [doi:10.1088/1126-6708/2006/05/026].

12. CMS Collaboration, CMS PAS QCD-10-004.

13. F. W. Bopp, R. Engel and J. Ranft, arXiv:hep$\mathrm{ph} / 9803437$.

14. CMS Collaboration, CMS PAS QCD-10-002.

15. V. Khachatryan et al. [CMS Collaboration], Phys. Rev. Lett. $\mathbf{1 0 5}$ (2010) 032001 [doi:10.1103/PhysRevLett.105.032001]. 